\section{Characterisation of electromagnetic compatibility drifts of nanoscale integrated circuit after accelerated life tests}

\section{S. Ben Dhia, A. Boyer, B. Li and A. Cisse Ndoye}

Presented is an original study about the effects of integrated circuit aging on electromagnetic emission and immunity to radio frequency interferences. For the first time an electromagnetic compatibility (EMC) qualification procedure is proposed to quantify the EMC level variation over the full lifetime of a component. Results presented show non-negligible variations of the emission and immunity thresholds after accelerated life tests, which could seriously deteriorate EMC margins required to ensure compliance with standard EMC levels.

Introduction: Owing to increasing usage of high speed and complex electronic systems, electromagnetic compatibility (EMC) is a major concern for electronic system manufacturers [1]. Ensuring EMC over the full lifetime of a product is a requirement for safety critical applications which bear harsh environments, e.g. for the automotive and the aerospace industries. However, the objective of long-term reliability is less and less achievable owing to the drastic reduction of device lifetime observed in nanometric CMOS technologies [2]. The natural aging, which accelerates degradation mechanisms in ICs, can also degrade the EMC performance of ICs [3-5]. However, its impact on EMC has not been completely clarified yet. EMC qualification tests are systematically realised on burn-in components and seldom combined with accelerated life and robustness tests. To verify if the degradation mechanisms induced by natural aging could affect component EMC performance, this Letter proposes an original study of the emission and susceptibility of a $65 \mathrm{~nm}$ CMOS IC before and after two standard accelerated life tests. Results presented underline emission spectrum drifts and immunity level deterioration after accelerated aging tests.

Test chip and experimental setup description: The tests are based on a $65 \mathrm{~nm}$ low power CMOS technology test chip developed by STMicroelectronics and dedicated to characterisation of several I/O structures. To exhibit aging effect on the test chip EMC, two standard measurement methods adapted to IC conducted emission and immunity characterisation are applied on two batches of five components before and after two different accelerated life tests. The number of tested samples results from a compromise between the level of confidence in measurements and EMC test duration and cost. The conducted emission of the component under test is characterised according to the standard IEC 61967-4 [6]. The parasitic current produced by the IC and flowing through the ground pin is sensed. The conducted susceptibility of this component is tested according to the IEC 62132-3 direct power injection (DPI) method [7]. DPI tests are conducted on a digital input and power supply pins. Accelerated life tests consist in applying stress conditions to devices in order to accelerate the internal degradations and then estimate device lifetime. High temperature operating life (HTOL) $\left(150^{\circ} \mathrm{C}\right.$ during $\left.408 \mathrm{~h}\right)$ and low temperature operating life (LTOL) $\left(-40^{\circ} \mathrm{C}\right.$ during $\left.408 \mathrm{~h}\right)$ [8] combined with high supply voltage (nominal $+10 \%)$ are selected.
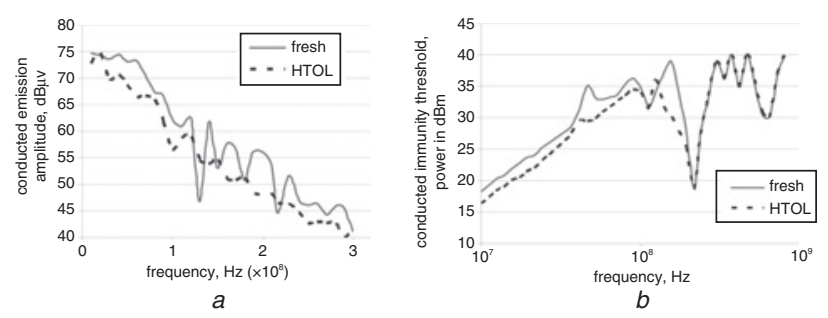

Fig. 1 Examples of EMC performances drift after accelerated aging tests $a$ HTOL aging impact on emission level (measured on one sample) $b$ HTOL aging impact on IO immunity threshold (RFI injected on IO power supply of one sample)

Experimental results: Fig. 1 presents two examples of EMC level variations after the HTOL test. For both results, a global reduction of emission spectrum as well as a reduction of the IC immunity threshold over a wide frequency range can be noticed. At some frequencies, the maximum drift reaches almost $8 \mathrm{~dB}$. These measurements are then performed on the five samples in order to quantify the drift trends statistically. Variations of emission or susceptibility levels are given in terms of mean drift $\Delta_{\mathrm{M}}$ over all the components:

$$
\Delta_{M}=\frac{1}{N_{\text {sample }}} \sum_{i=1}^{N}\left(X_{A i}-X_{B i}\right)
$$

$X_{B i},\left(X_{A i}\right)$ : emission or immunity level of sample 'i’ before (resp. after) aging; $N_{\text {sample }}$ : number of samples.

Emission and susceptibility levels cannot be accurately known as they are subject to statistical distributions due to measurement errors and variability between components. Aging can affect the variability of component characteristics so the dispersion $\sigma$ of emission or susceptibility levels must be computed before and after aging:

$$
\sigma=\sqrt{\frac{\sum_{i=1}^{N}\left(X_{(A \circ \mathrm{or} B) i}-\overline{\left.X_{(A \circ \mathrm{or} B}\right)}\right)^{2}}{N_{\text {sample }}-1}}
$$

$\bar{X}$ : mean emission or immunity level of all samples.

The measured drifts between samples are also affected by repeatability errors owing to the measurement equipment or test bench variations. Characterisation shows that this error remains lower than $0.4 \mathrm{~dB}$ for emission measurements and $0.12 \mathrm{~dB}$ for immunity measurements. As systematic errors due to equipment uncertainties are independent of components, they do not affect the aging induced drift measurement.

Emission level drifts analysis: Fig. $2 a$ shows the mean difference between the emission levels of fresh and aged components, for both accelerated life tests, also summarised in Table 1. Emission levels decrease clearly after both aging tests over almost all the frequency range. Low temperature stress induces a greater emission level reduction (with a mean value about $-1.48 \mathrm{~dB}$ ) than high temperature stres ( $-0.3 \mathrm{~dB}$ on average). A reduction of emission level can be explained by a decrease of transient currents induced by IC activity, which results from internal degradation mechanisms. The results in Fig. $2 b$ show that emission levels tend to spread out after both accelerated life tests (mean of $0.4 \mathrm{~dB}$ ), although it remains within an acceptable margin.
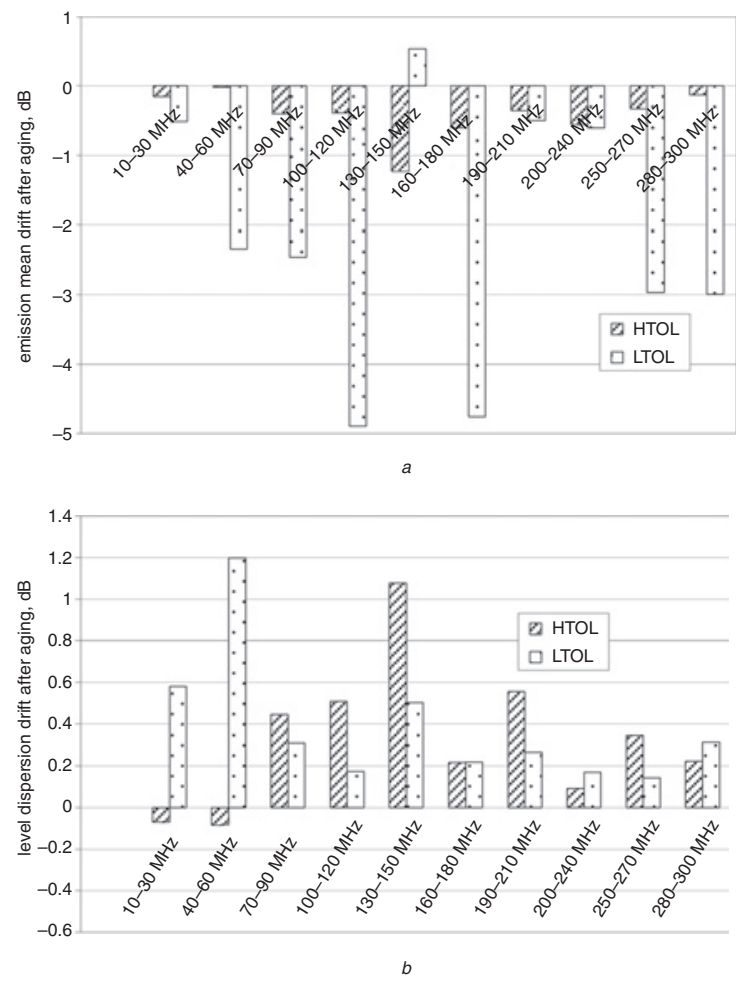

Fig. 2 Overview of components emission drifts after accelerated life tests $a$ LTOL and HTOL aging impact on emission level (mean drift for all samples) $b$ LTOL and HTOL aging impact on emission level dispersion between samples (difference between aged and fresh dispersion) 
Table 1: Emission and immunity results summary

\begin{tabular}{|c|c|c|c|c|c|c|}
\hline \multirow[t]{2}{*}{ EMC test } & \multirow[t]{2}{*}{ Aging test } & \multirow[t]{2}{*}{$\begin{array}{l}\text { Max. aging } \\
\text { drift above all } \\
\text { frequencies } \\
\text { (for most } \\
\text { affected } \\
\text { sample) } \\
\text { (dB/dB) } \\
\end{array}$} & \multirow[t]{2}{*}{$\begin{array}{c}\text { Mean aging } \\
\text { drift above } \\
\text { all frequencies } \\
\text { (for most affected } \\
\text { sample) (dB) }\end{array}$} & \multirow[t]{2}{*}{$\begin{array}{c}\text { Mean aging } \\
\text { drift for } \\
\text { batch above all } \\
\text { frequencies (dB) }\end{array}$} & \multicolumn{2}{|c|}{$\begin{array}{l}\text { Standard deviation } \\
\text { of drift for batch } \\
\text { above all } \\
\text { frequencies }\end{array}$} \\
\hline & & & & & $\begin{array}{l}\sigma \text { (standard } \\
\text { deviation) } \\
(\mathrm{dB})\end{array}$ & $\begin{array}{c}\text { Expanded } \\
\text { uncertainty } \\
\mathrm{k} \sigma=2.78 \mathrm{x} \sigma \\
(\mathrm{dB})\end{array}$ \\
\hline \multirow{2}{*}{$\begin{array}{l}\text { Conducted } \\
\text { emission }\end{array}$} & HTOL & $2.2 /-9.5$ & -0.8 & -0.3 & 1 & 2.78 \\
\hline & LTOL & $15.3 /-12$ & -2.9 & -1.48 & 1 & 2.78 \\
\hline \multirow{2}{*}{$\begin{array}{l}\text { IO immunity } \\
\text { (RFI on IO) }\end{array}$} & HTOL & $1 /-0.5$ & 0.3 & 0.5 & 0.1 & 0.278 \\
\hline & LTOL & $12.8 /-12.5$ & 2.4 & 0.83 & 1.5 & 4.17 \\
\hline \multirow{2}{*}{$\begin{array}{l}\text { IO immunity } \\
\text { dB (RFI on } \\
\text { IO supply) }\end{array}$} & HTOL & $2 /-8.75$ & -1.6 & -0.8 & 0.7 & 1.9 \\
\hline & LTOL & $1.26 /-8.25$ & -0.5 & -0.6 & 1 & 2.78 \\
\hline
\end{tabular}

Immunity drifts analysis: Results shown in Fig. $3 a$ compare HTOL and LTOL aging impact on the IO immunity for an RF injection on the IO input. HTOL tends to enhance slightly the immunity level over a large frequency range. However, LTOL seems to have an ambiguous impact on susceptibility level which can strongly increase or decrease depending on the frequency range. Nevertheless, the mean drift of susceptibility level for the entire batch remains positive for both aging tests, leading us to think that aging has a positive but weak effect on IO immunity during conducted injection on input pins. Conversely, aging seems to have a negative impact on IO immunity when RF injections are performed on power supply pins. As shown in Fig. $3 b$ and Table 1, HTOL and LTOL tests induce a noticeable reduction of the immunity level of the IO over a large frequency range. Even if the average global fading of immunity level remains acceptable compared to other sources of level dispersion, the large reductions of immunity level observed on some samples at several frequencies (up to $-8 \mathrm{~dB}$ ) can seriously affect their EMC compliance. Some investigations on IO characteristics have been made before and after aging (e.g. input-output transfer function, I/V characteristics). Even if they show slight variations, they are hardly correlated to susceptibility level variations. Further analyses on I/O internal structures are required to explain in more detail the effects of IO aging on immunity level degradation.
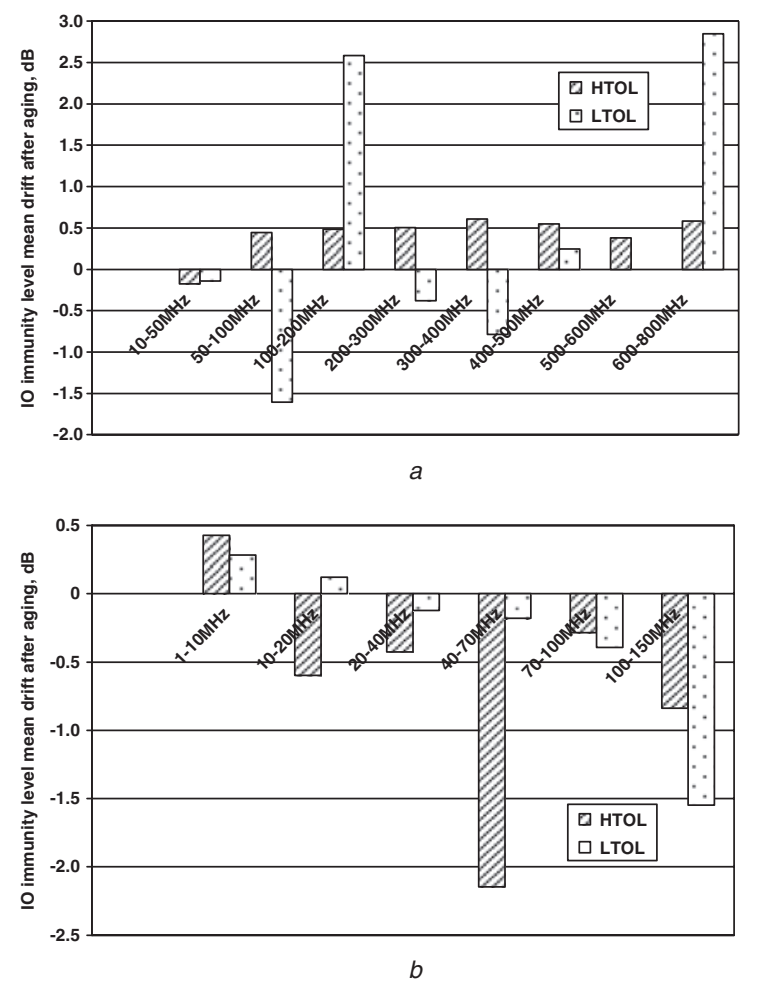

Fig. 3 Overview of IO and power supply immunity threshold drifts after accelerated life tests

$a$ Comparison between HTOL and LTOL aging impact on IO immunity (RFI injected on IO input): mean drift for all samples

$b$ Comparison between HTOL and LTOL aging impact on IO immunity (RFI

injected on IO supply): mean drift for all samples
Conclusion: Although the consequences of integrated circuits' natural aging on electromagnetic emission and susceptibility could have a dramatic impact on electronic system functional safety, they have not yet been evaluated. This Letter shows through a case study the non-negligible effect of component aging on EMC levels. The negative impact of aging on component susceptibility to radio frequency interferences raises an important issue to be solved to warrantee electronic system reliability throughout their exploitation duration.

Acknowledgments: The authors thank L. Courau and M. Fer from STMicroelectronics, Crolles, France, for material support.

(C) The Institution of Engineering and Technology 2010

27 October 2009

doi: $10.1049 / \mathrm{el} .2010 .2885$

S. Ben Dhia, A. Boyer, B. Li and A. Cisse Ndoye (Department of Electronics, INSA de Toulouse, 135 avenue de rangueil, Toulouse 31077, France)

E-mail: sonia.bendhia@insa-toulouse.fr

\section{References}

1 Ben Dhia, S., Ramdani, M., and Sicard, E.: 'Electromagnetic compatibility of integrated circuits' (Springer, ISBN 0-387-26600-3), 2005

2 Srinivasan, J., Adve, S.V., and Bose, P.: 'The impact of technology scaling on lifetime reliability'. Int. Conf. on Dependable Systems and Networks, Florence, Italy, June 2004

3 Parker, W.H. and Tustin, W.: 'The case for combining EMC and environmental testing', ITEM 2002, pp. 54-60, 2002

4 Ben Dhia, S., Ndoye, A.C., Boyer, A., Guillot, L., and Vrignon, B.: 'IC emission spectrum drifts after burn-in cycles'. 2008 Asia Pacific EMC Symp., Singapore, May 2008

5 Fernandez, R., Rodriguez, R., Nafria, M., and Aymerich, X.: 'Effect of oxide breakdown on RS latches', Microelectron. Reliab., 2007, 47, pp. $581-584$

6 IEC 61967-4, 'Integrated circuits, measurement of electromagnetic emissions, $150 \mathrm{KHz}-1 \mathrm{GHz}$ : Measurement of conducted emissions, $1 \Omega / 150 \Omega$ method', IEC standard, 2006

7 IEC 62132-3, 'Direct RF power injection to measure the immunity against conducted RF-disturbances of integrated circuits up to $1 \mathrm{GHz}$, IEC standard, 2007

8 Automotive Electronics Council, Component Technical Committee, Stress test qualification for integrated circuits, AEC-Q100-Rev-F, 2003 